International Journal of Wireless \& Mobile Networks (IJWMN) Vol. 4, No. 1, February 2012

\title{
Improvement of LTE System Performances by Using a New Pilot Structure
}

\author{
H. Chamkhia, A. Omri and R. Bouallegue \\ Innov'Com laboratory, Tunisia
}

\begin{abstract}
In this paper, a new pilot structure for LTE downlink system in SISO and MIMO cases is introduced in order to improve the channel estimation performances. The downlink reference signal structure in LTE is important for channel estimation. Pre-defined resource elements in the time and frequency domain are carrying the cell-specific reference signal sequence. However, those standard pilot scattering contains more empty resource element as the number of transmit or receiver antennas increases, leading to a decrease of the system performances. Simulation results shows that the proposed LTE pilot structure allows a high performance in terms of QoS and throughput for both SISO and MIMO cases.
\end{abstract}

Index Terms - LTE, Pilot Scattering, SISO, MIMO-OFDM, BER.

\section{Introduction}

Aiming to improve cellular radio communication and to shape the evolution of the current UMTS system, many organizations such as 3GPP have proposed different trials of standardization. The current proposal system also known as 3GPP- Long-Term Evolution (LTE) is a step towards the fourth generation (4G) mobile radio technologies to increase capacity and speed of mobile telephone networks [1].

The first version of LTE is proposed in Release 8 of the 3GPP specifications [2]. OFDMA (Orthogonal Frequency Division Multiple Access) is the modulation used in this standard for the downlink and SC-FDMA (Single Carrier Frequency Division Multiple Access) is the modulation used for the uplink [1]. The downlink transmission model for E-UTRA is based on MIMO-OFDM [2]. In an OFDM system, the available spectrum is divided into multiple mutually orthogonal carriers, called sub-carriers. Each one of those sub-carriers is independently modulated by a low rate data stream [3]. OFDM is a robust modulation against multipath fading, however, it is highly sensitive to the Doppler shift which destroy the subcarrier orthogonality and give rise to intercarrier interference (ICI) [4]. Aiming to overcome those ICI in LTE system, several channel estimation techniques have been proposed $[5,6,7,8,9]$. To facilitate the estimation of the channel in an OFDM system, known signals or pilots could be inserted in the transmitted OFDM symbol. 
International Journal of Wireless \& Mobile Networks (IJWMN) Vol. 4, No. 1, February 2012

In LTE system, the pilot structure has a static pattern as defined in the Release 8 for both SISO and MIMO structure. Since pilots are useful for synchronization and channel estimation, the system has to guarantee the well reception of those informations despite the existing interferences resulting from simultaneous multiple transmissions[10]. In this order, the standard LTE pilot pattern is designed with specific resource elements for pilot transmission. However, those resource elements can be left empty allowing other antennas to transmit their pilot [1]. Consequently, this standard structure seems not optimal in terms of throughput system since there are no uses of some resource elements

In this paper we present a new pilot scattering for LTE downlink system based on a DVB pilot pattern [11]. This new pilot scattering improve the system performances and correct the loss of resource elements.

The reminder of the paper is organized as follows: Section II provides the MIMO-OFDM transmission scheme. Analysis of standard LTE pilot scattering is introduced in section III. The improvement of LTE pilot structure is shown in section IV. Section V provides the numerical results and finally section VI concludes the paper.

\section{MIMO-OFDM transmission schemes}

In this section, the MIMO OFDM transmission scheme is described. The modulation block is used to modulate the original binary data symbol using the complex constellation QPSK, 16 QAM or 64..QAM according to the LTE standard [12, 13, 14]. Multiple antennas can be used at the transmitter and receiver, therefore multiple-input multiple-output (MIMO) encoders are needed to increase the spatial diversity or the channel capacity. The purposes of MIMO systems implementation are various: we can obtain either a diversity gain to combat signal fading or we can obtain a capacity gain. Generally, there are three types of MIMO receivers as presented in [15].The first improves the power efficiency by maximizing spatial diversity. The second is used to increase the capacity. Finally, the third exploits the knowledge of the channel at the transmitter.

LTE system is using Alamouti STBC coding which improves the power efficiency by maximizing spatial diversity. The OFDM modulation scheme consists of transmitting a block of information symbols in parallel on channel subcarriers. An OFDM modulator can be easily and efficiently implemented using the inverse discreet Fourier transform (IDFT) on a block of information symbols. Each block of IDFT coefficients is preceded by a cyclic prefix (CP) with length at least equal to the channel delay spread to prevent inter symbol interference (ISI) caused by multipath channel propagation [16]. Commonly, a pilot sequence insertion is used in the channel estimator to predict a refined channel frequency response at the receiver to equalize for the channel impairments and consequently to estimate the transmitted signal. The Fig. 1 summarizes the transmitter scheme as following: 
International Journal of Wireless \& Mobile Networks (IJWMN) Vol. 4, No. 1, February 2012

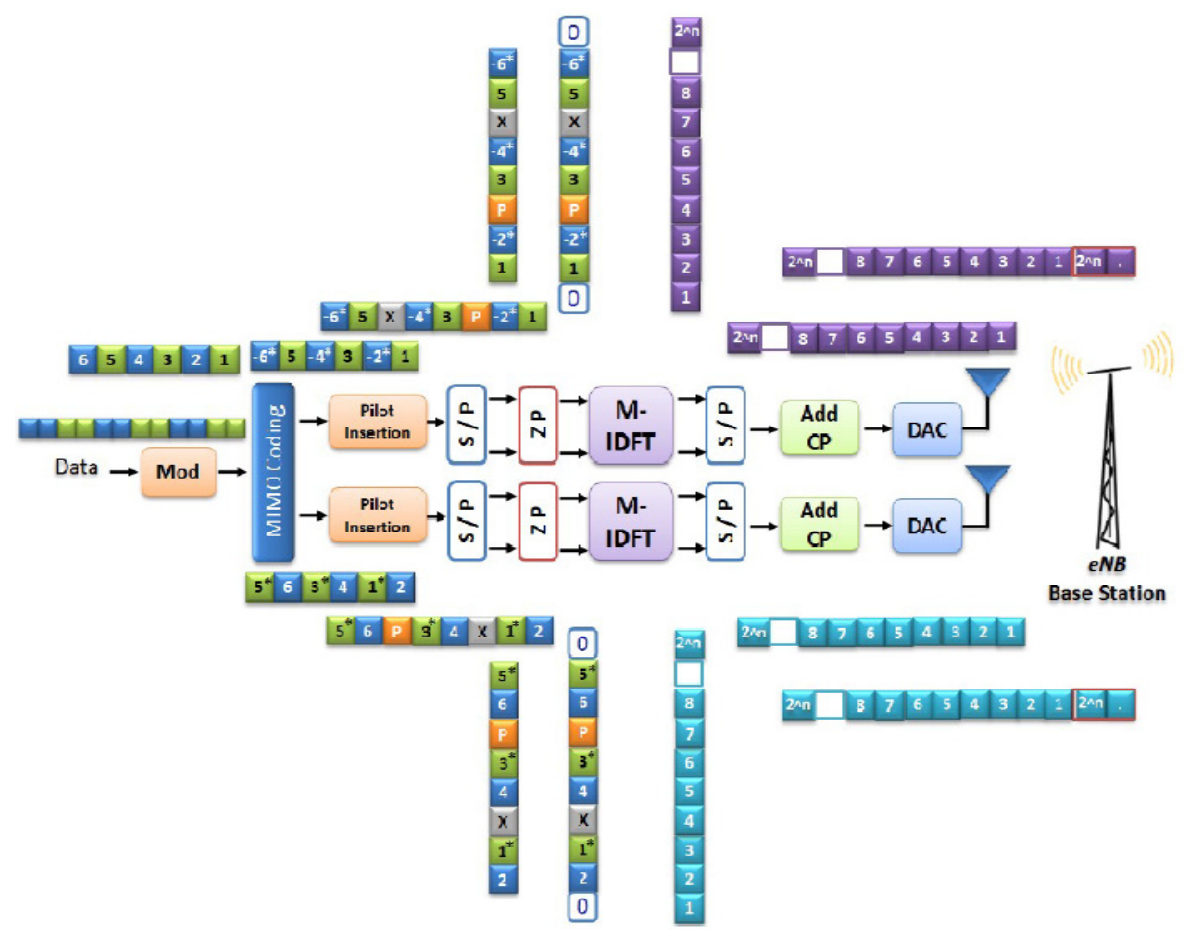

Figure 1: MIMO-OFDM transmission.

The receiver scheme is the inverse operations and it is shown in Fig. 2

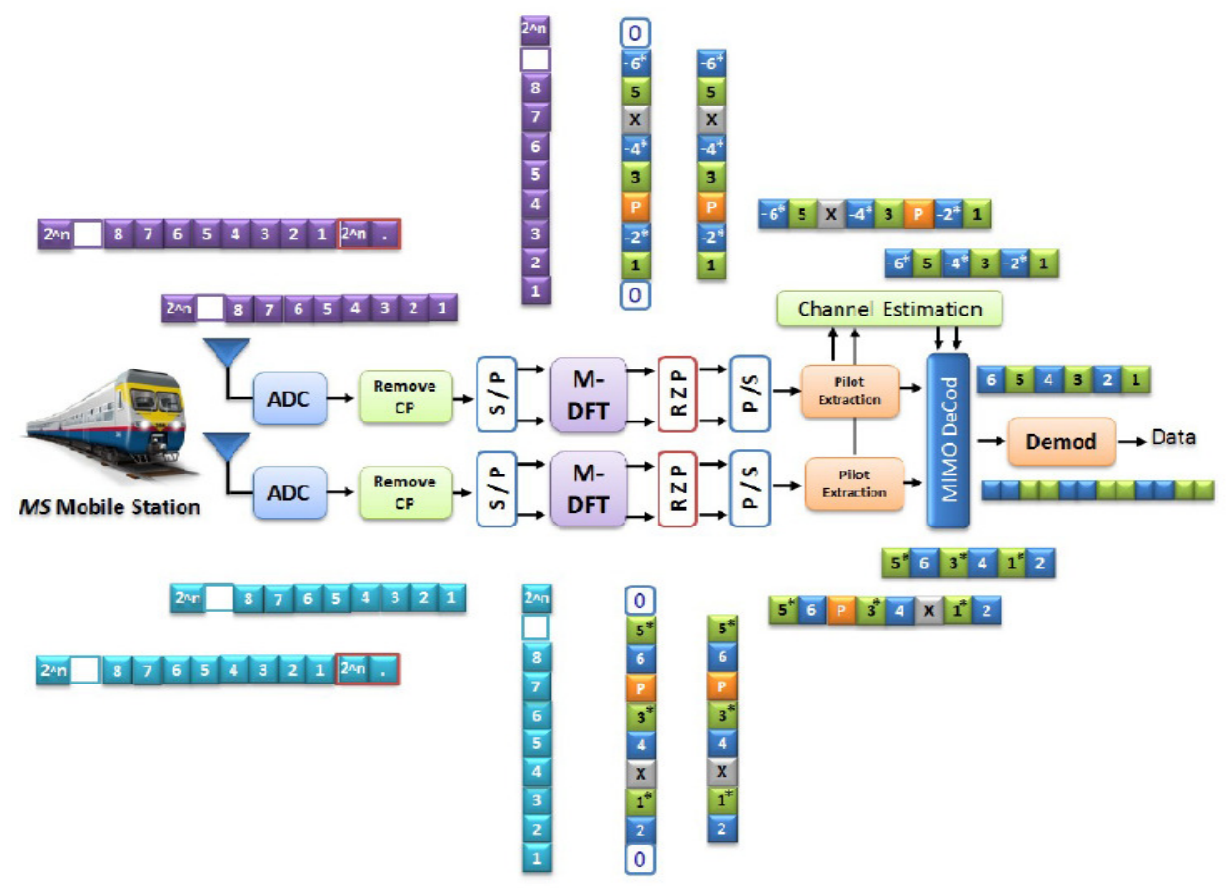

Figure 2: MIMO-OFDM reception. 
International Journal of Wireless \& Mobile Networks (IJWMN) Vol. 4, No. 1, February 2012

\section{Analysis of standard LTE pilot scattering}

The LTE DownLink reference signal structure is important for cell search, channel estimation and neighbor cell monitoring. the LTE standard offers a static pilot scattering mentioned in the following sections for both SISO and MIMO cases.

\subsection{SISO case}

In this section, the conventional pilot scattering of a SISO LTE system is presented. The SISO case uses 4 resource unit per resource block to inform about pilots. Reference signals are transmitted during the first and fifth OFDM symbols of each slot when the short cyclic prefix (CP) is used and during the first and fourth OFDM symbols when the long CP is used [1]. The frequency spacing between two successive reference signal is 6 subcarriers. The following figure illustrates the pilot scattering for this case:

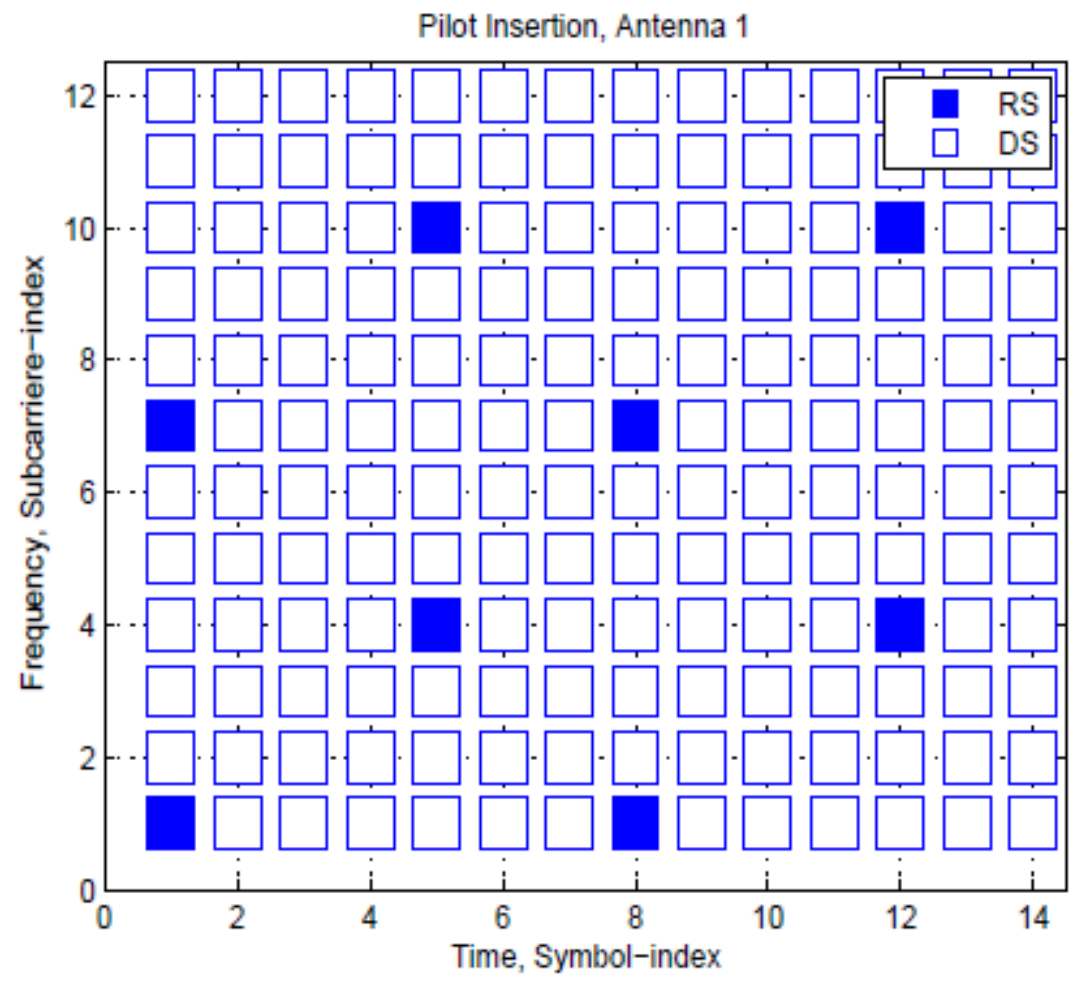

Figure 3: Pilot structure for SISO LTE system.

\subsection{MIMO case}

Fig. 4 shows how the time-frequency access works in a MIMO environment, that is one of the innovation introduced by LTE to reach higher data rates. In this case with two antennas, the pilots corresponding to the other antennas pilots have to be turned off (symbols in gray) in order to avoid interference [1]. 

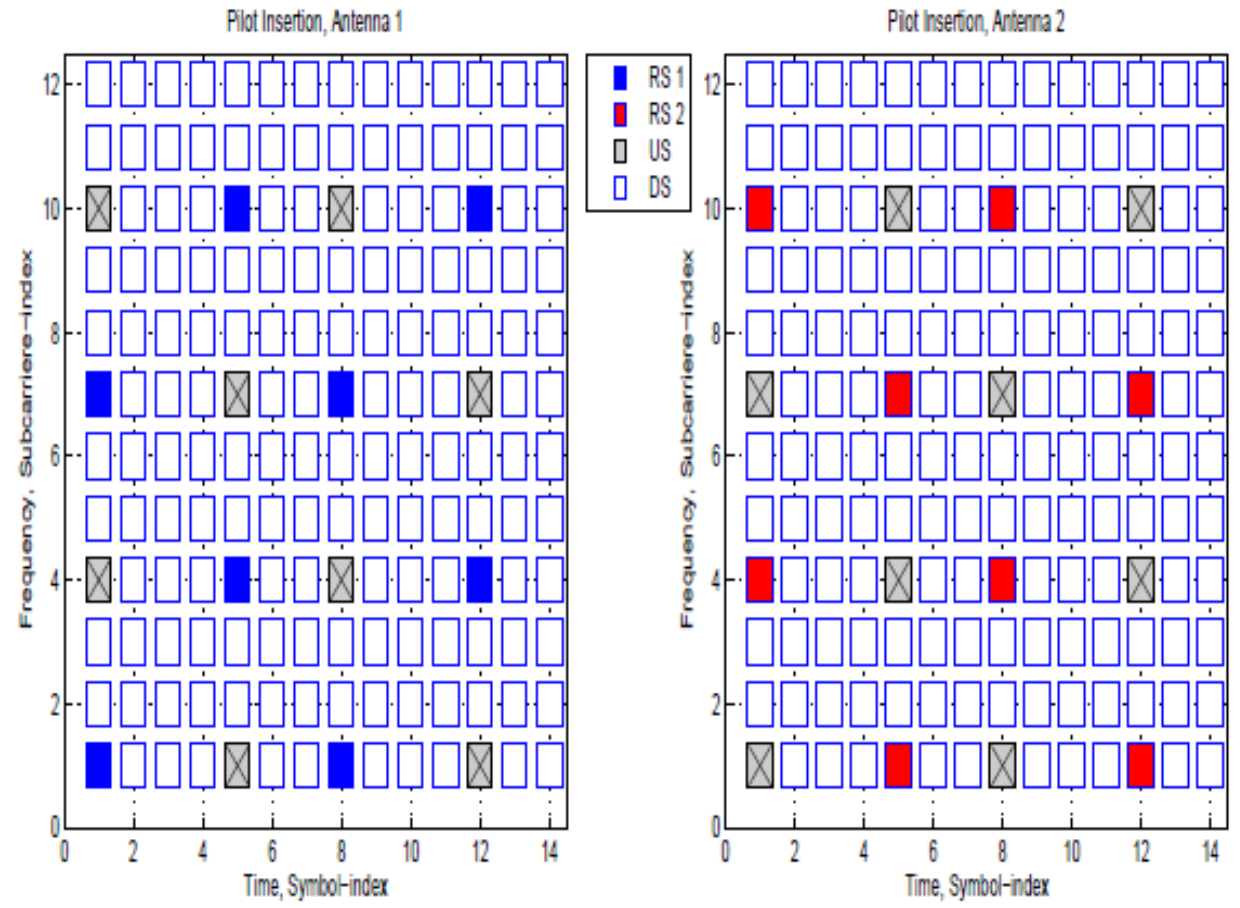

Figure 4: Pilot structure for MIMO LTE system.

\section{Improvement of LTE pilot structure}

The contribution of this paper is to propose a new optimized pilot pattern for an LTE system based on the DVB pilot disposition [11] in order to improve the system quality of services. The purpose is to use the DVB pilot pattern in an LTE system and compare the performance of the new pilot structure with the standard LTE pilot scattering. In the case of SISO, for both usual and proposed cases, there is no silent resource block as long as only one antenna is transmitting. For a MIMO transmission scheme, the performances of the new pattern are more scalable. In this section, we investigate the new pilot patterns for SISO and MIMO cases.

\subsection{New LTE pilot scattering in SISO case}

The location and amplitudes of the pilots differ with respect to the transmission parameters. It can depending on the FFT size, the length of the guard interval, pilot pattern and the antenna settings. The contribution here is to define a new disposition for the pilot which help the system to improve the quality.

Fig. 5 shows the locations of scattered and other pilots for a sample pilot pattern. The edge pilots are inserted in order to allow frequency interpolation up to the edge of the spectrum. Scattered pilots are inserted in different symbols, which may carry data and are used to transmit signaling information. 
International Journal of Wireless \& Mobile Networks (IJWMN) Vol. 4, No. 1, February 2012

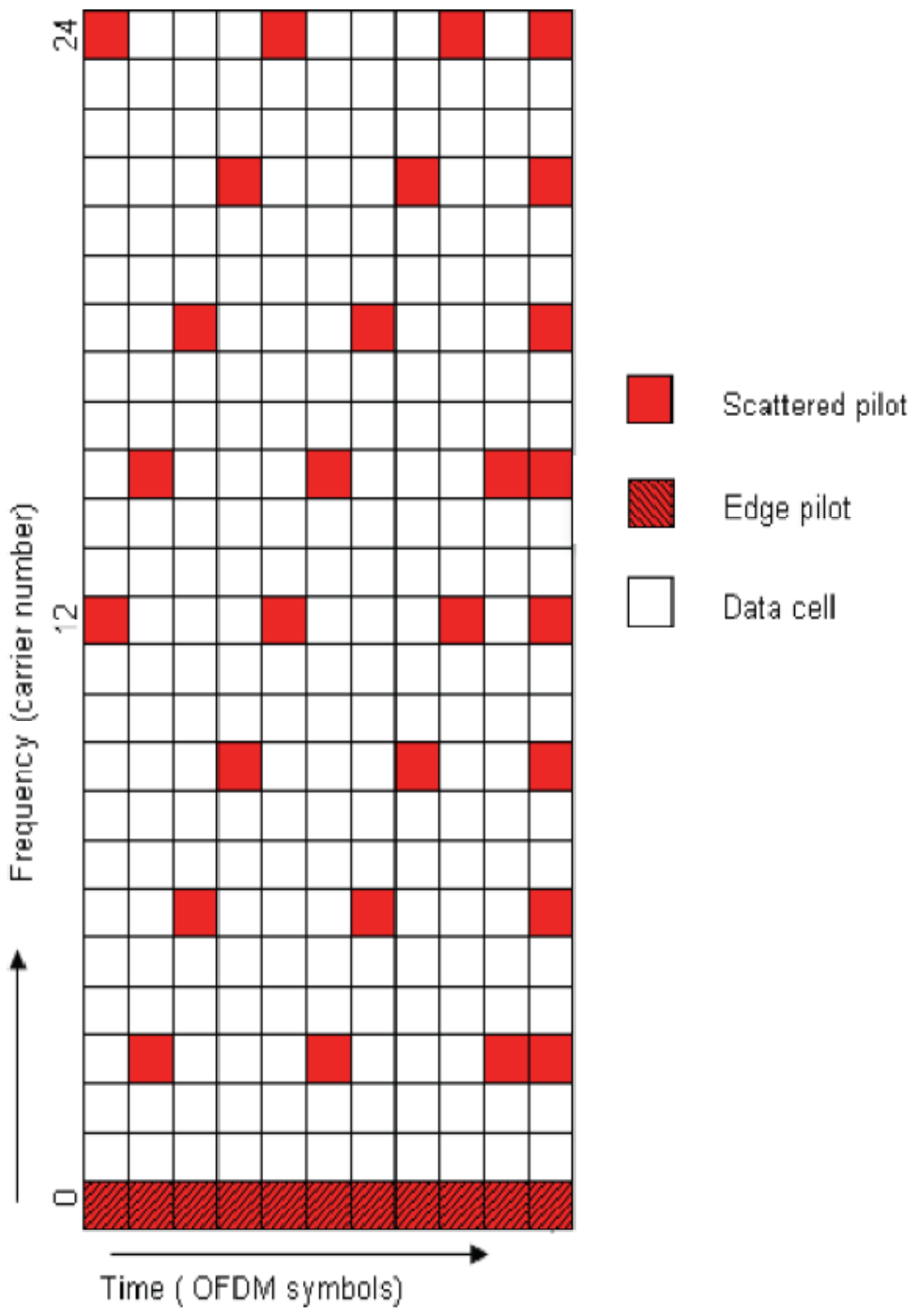

Figure 5: New LTE scattered pilot pattern for SISO transmission.

\subsection{New LTE pilot scattering in MIMO case}

Fig. 6 shows the pilot location for a 2x2 MIMO transmission case. The pilot transmission is proceeded in two successive time slot where the channel is supposed fix: In the first time slot, the first and the second antennas send their reference signal $(R S)$ (or pilots) respectively named $P 1$ and $P 2$.

In the second time slot, the pilot resource element of the first antenna carry its pilot P1, however the pilot resource element of the second antenna carry $-P 2$.

The information of the first time slot sent from both transmit antennas is added providing the receivers with $(P 1+P 2)$. The same procedure is done in the second time slot providing the receivers with $(P 1-P 2)$. Having the aforementioned expression, receivers can deduce pilot of both transmit antennas. 
International Journal of Wireless \& Mobile Networks (IJWMN) Vol. 4, No. 1, February 2012
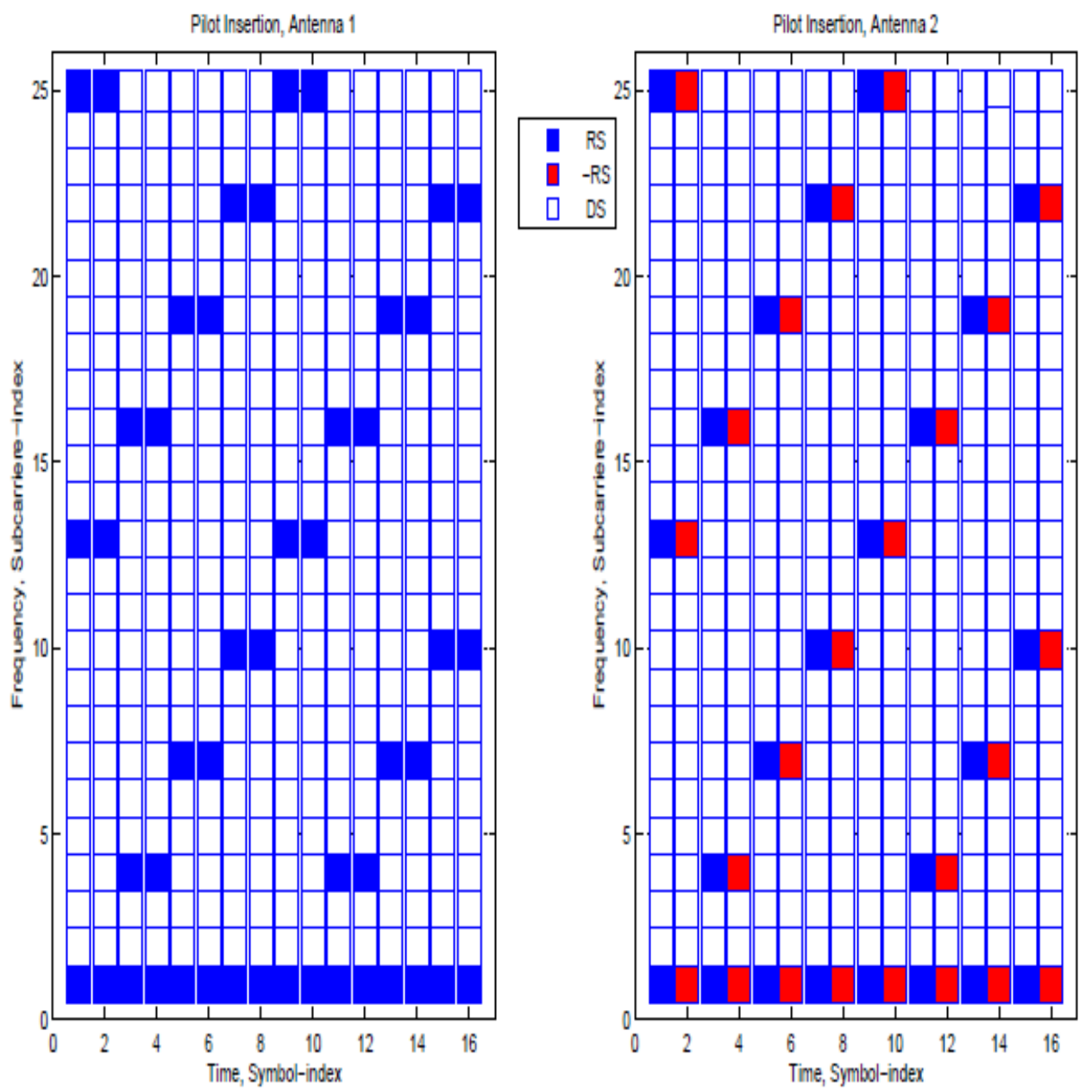

Figure 6: New LTE scattered pilot pattern for MIMO transmission.

\section{Numerical results}

In this section, we present simulation results to verify the performances of the new LTE pilot pattern presented. First, we define the simulation parameters and later the results are discussed in details.

\subsection{Simulation setup}

We are investigating the BER of the system in the cases of SISO and MIMO transmission. All the simulation results are generated by varying the average SNR and using a polynomial interpolation method to estimate the channel. OFDM System is simulated using the parameters shown in Table $1[2,10,17,18]$. These parameters are based on downLink LTE system. 
International Journal of Wireless \& Mobile Networks (IJWMN) Vol. 4, No. 1, February 2012

Table 1: Simulation Parameters

\begin{tabular}{|c|c|}
\hline Parameters & Specifications \\
\hline OFDM system & LTE \\
\hline Bandwidth(MHz) & 20 \\
\hline Constellation & $16-\mathrm{QAM}$ \\
\hline Mobile Speed & $50 \mathrm{Km} / \mathrm{h}$ \\
\hline Frequency(GHz) & 2 \\
\hline Channel Type & EVA(Extended Vehicular A) \\
\hline Channel Estimation Method & Polynomial Interpolation \\
\hline
\end{tabular}

\subsection{Discussion}

Fig.7 shows the variation of BER as a function of $\mathrm{E}_{\mathrm{s}} / \mathrm{N}_{0}$ in a SISO case. Noticeably, the proposed patten outperforms the standard pilot scattering of LTE and gives a results closer to the known channel evaluation. For example at a BER $=10^{-2}$, a gain of $7 d B$ over the standard pattern is obtained. As shown in Fig. 8, the performance of the introduced contribution in MIMO case outperforms the standard LTE MIMO pilot scattering in terms of BER and in term of data transmission speed. This can be explained by the use of less number of LTE pilot in the new contribution compared to the standard pattern.

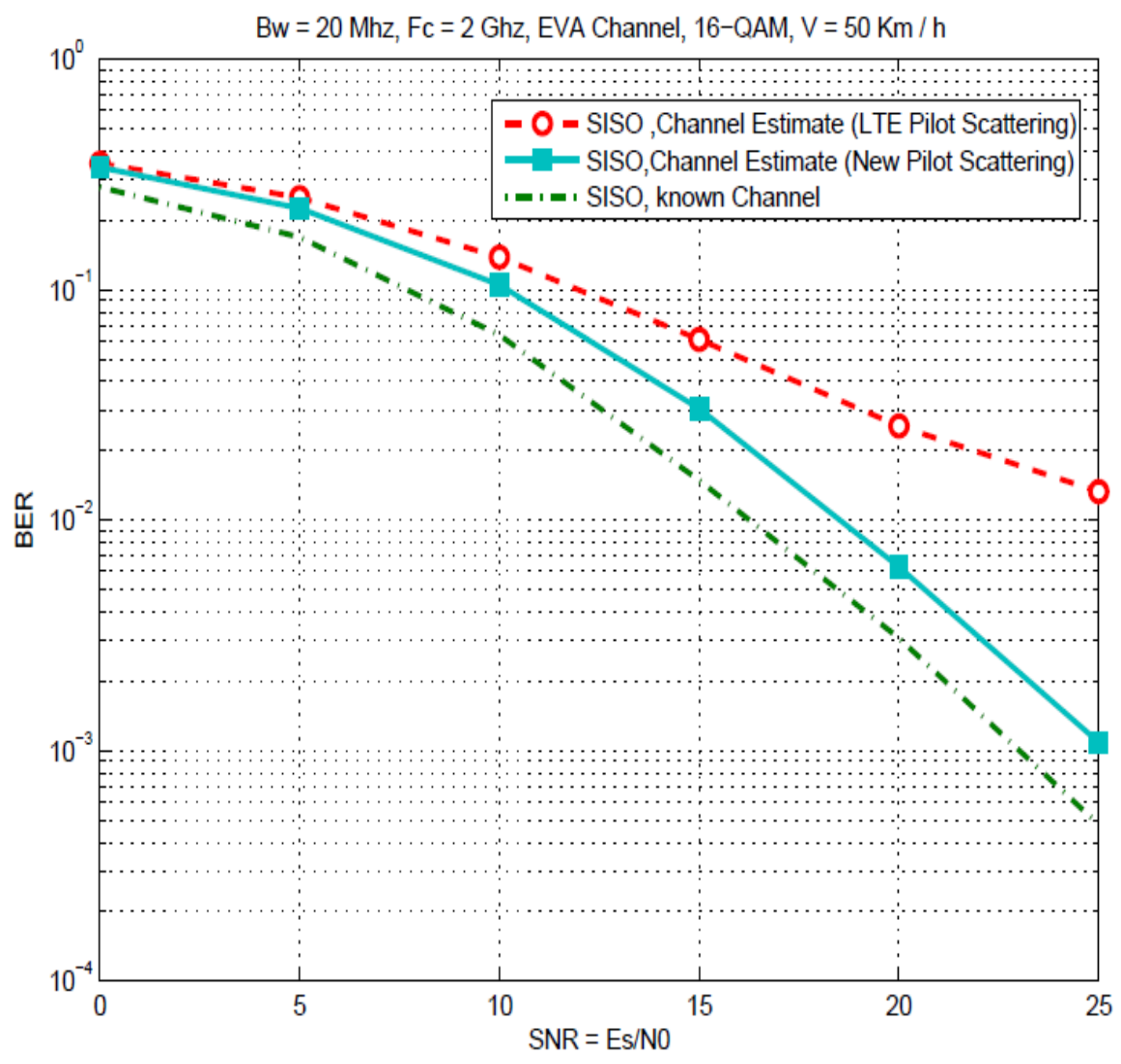

Figure 7: BER vs SNR. 


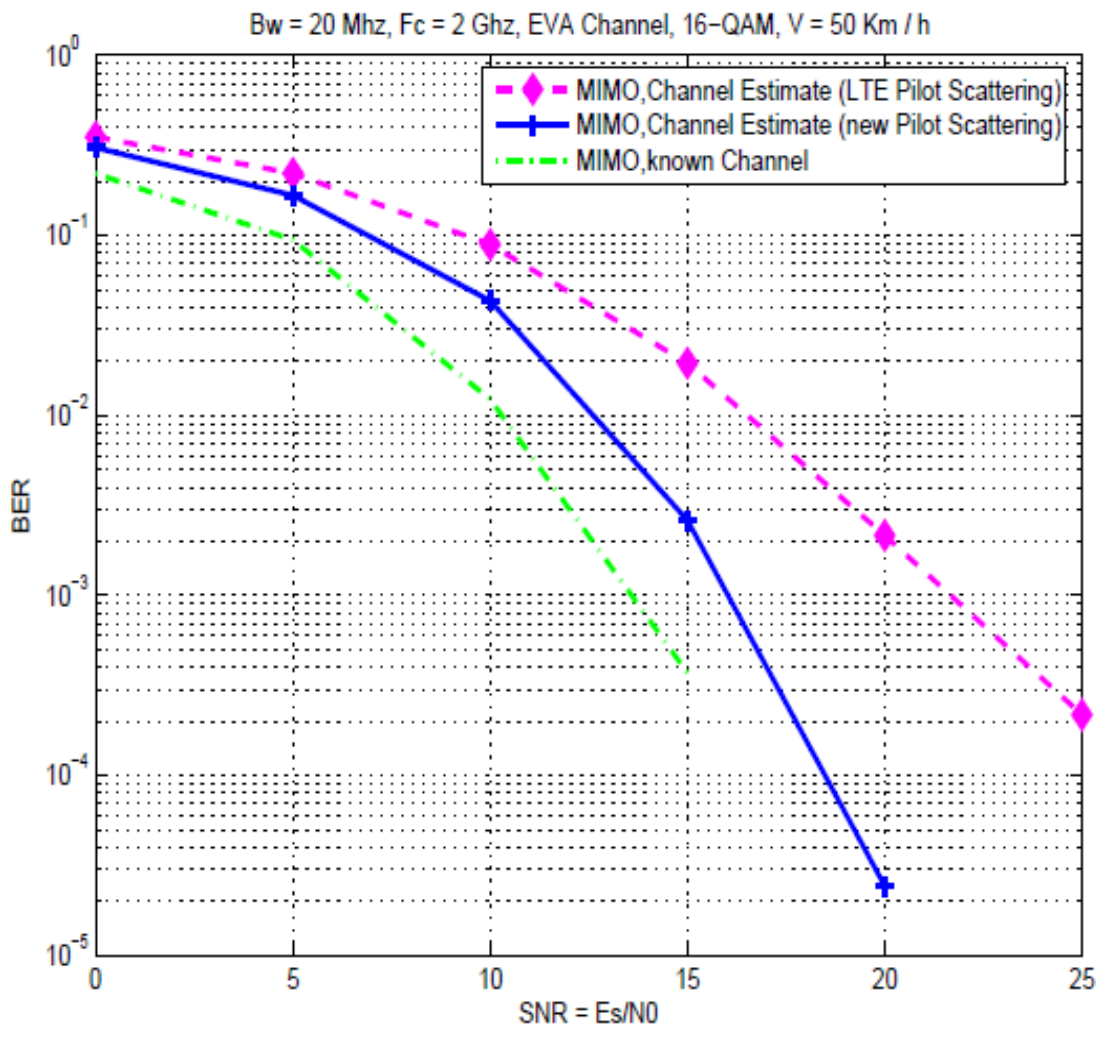

Figure 8: BER vs SNR.

\section{Conclusion}

Pilots are important information for the LTE system since they are primary elements for synchronization and channel estimation. However, pilot structure given by standard pattern can badly affect evaluative criteria of the system such as throughput and QoS. In this paper, we have introduced a new pilot structure for LTE downlink system based on the DVB structure.

MIMO and SISO cases were studied using the new pilot pattern and based on 3GPP specifications. Simulation results confirm the significant performance improvement that can be achieved when compared to the standard LTE pilot structure. This new LTE pilot structure allows a high performance in terms of QoS and in terms of throughput for the MIMO cases since the introduced pilot structure used less number of pilot then the standard LTE pilot structure.

\section{References}

[1] M. Rumney, LTE and the Evolution to 4G Wireless Design and Measurement Challenges, pp. 555567, Agilent Technologies Publication, 2009. 
International Journal of Wireless \& Mobile Networks (IJWMN) Vol. 4, No. 1, February 2012

[2] 3rd Generation Partnership Project, Technical Specification Group Radio Access Network; evolved Universal Terrestrial Radio Access (UTRA): Physical Channels and Modulation layer, pp. 55 - 67, TS 36.211, V8.8.0, 2009.

[3] J. Ketonen, M. Juntti, and J. R. Cavallaro, "Performance complexity comparison of receivers for a mimo-ofdm system," IEEE Transaction on Signal Processing, vol. 58, pp. 3360-3372, 2010.

[4] R. Van Nee and R. Prasad, OFDM forWireless Multimedia Communications, pp. 1-11, 2000.

[5] C. Lim and D. Han, "Robust ls channel estimation with phase rotation for single frequency network in ofdm," IEEE Transactions on Consumer Electronics, vol. 52, pp. 1173-1178, 2006.

[6] S. Galih, T.Adiono, and A.Kurniawan, "Low complexity mmse channel estimation by weight matrix elements sampling for downlink ofdma mobile wimax system," International Journal of Computer Science and Network Security b (IJCSNS), vol. 528, pp. 1173-1178, 2010.

[7] A. Baynast, A. Sabharwal, and B. Aazhang, "Analysis of decision-feedback based broadband ofdm systems," Conference on Signals Systems and Computers ACSSC, vol. 8, pp. 73-80, 2005.

[8] E.K. Hlel, S. Cherif, F. Tlili, and M. Siala, "Improved estimation of time varying and frequency selective channel for ofdm systems," ICECS, vol. 1, pp. 11-77, 2008.

[9] E. K. Hlel, S. Cherif, F. Tlili, and M. Siala, "Improved estimation of time varying and frequency selective channel for ofdm systems," ICECS, vol. 11, pp. 11-111, 2005.

[10] 3rd Generation Partnership Project, Technical Specification Group Radio Access Network; Physical layer aspects for evolved Universal Terrestrial Radio Access (UTRA), pp. 11 - 22, TR 25.814, V7.1.0, 2006.

[11] ETSI EN 300 744, Digital Video Broadcasting (DVB); Framing structure, channel coding and modulation for digital terrestrial television, V 1.6.1, 2009.

[12] Z. Lin, P. Xiao, B. Vucetic, and M. Sellathurai, "Analysis of receiver algorithms for lte scfdma based uplink systems," IEEE Transaction on Wireless Communications, vol. 9, pp.60-65, 2010.

[13] I. Khan and P. S. Hall, "Experimental evaluation of mimo capacity and correlation for narrowband body-centric wireless channels," IEEE Transaction on Antennas and Propagation, vol. 58, pp. 195202, 2010.

[14] J. F. ValenzuelaValdes, M. A. Garcia Fernandez, A. M. Martinez Gonzalez, and D. A.SanchezHernandez, "Evaluation of true polarization diversity for mimo systems," IEEE Transaction on Antennas and Propagation, vol. 58, pp. 2746-2755, 2009.

[15] C. Meng and J.Tuqan, "Precodedstbc-vblast for mimo wireless communication systems," IEEE International Conference on Acoustics, Speech and Signal Processing ICASSP, vol. 3, pp. 337-340, 2007.

[16] A. Dweikand, R. Hamila, and M. Renfors, "Blind estimation of large frequency offset in wireless ofdm systems," IEEE Transactions on Vehicular Technology, vol. 56, pp. 965-968, 2007.

[17] 3rd Generation Partnership Project, Technical Specification Group Radio Access Network; evolved Universal Terrestrial Radio Access(UTRA): Base Station (BS) radio transmission and reception, pp. 22 - 33, TS 36.104, V8.7.0, 2009.

[18] 3rd Generation Partnership Project, Evolved Universal Terrestrial Radio Access (E-UTRA); User Equipment (UE) radio transmission and reception, pp. 22 - 33, ARIB STD-T63-36.101, V8.4.0, 2008. 